CERN-TH/97-259

\title{
Supersymmetric Q-balls as dark matter
}

\author{
Alexander Kusenkof and Mikhail Shaposhnikov* \\ Theory Division, CERN, CH-1211 Geneva 23, Switzerland
}

\begin{abstract}
Supersymmetric extensions of the standard model generically contain stable non-topological solitons, Q-balls, which carry baryon or lepton number. We show that large Q-balls can be copiously produced in the early universe, can survive until the present time, and can contribute to dark matter.
\end{abstract}

CERN-TH/97-259

September, 1997

* email address: Alexander.Kusenko@cern.ch

** email address: mshaposh@nxth04.cern.ch 


\section{Introduction}

The nature of the missing matter in the universe remains one of the most intriguing outstanding problems in particle physics and cosmology. Stable particles hypothesized in theories beyond the standard model have been considered as candidates for dark matter. In the supersymmetric extensions of the standard model, the lightest supersymmetric particle (LSP), whose stability may be guaranteed by the conservation of R-parity, can naturally contribute to the hidden mass.

Recently, it has been pointed out that non-topological solitons, Q-balls [1, 2], are generically present in the MSSM [3]. Q-ball is a coherent state of a complex scalar field, whose existence and stability are due to the conservation of some global U(1) quantum number. In the MSSM the usual baryon and lepton numbers may play the role of such conserved quantity for the Q-balls built of squarks and sleptons, respectively. One can ask, whether these objects could have formed in the early universe, and whether their lifetime can be long enough for them to survive until present and contribute to dark matter.

Several mechanisms could have lead to the formation of Q-balls in the early universe. Non-topological solitons can be created [4 in the course of a phase transition ("solitogenesis"), or they can be produced via fusion [5, 6, 7] in a process reminiscent of the big bang nucleosynthesis ("solitosynthesis"). Finally, small Q-balls [8] can be pair-produced at high temperature. None of these scenarios, however, seems to be capable of producing large enough Q-balls to survive the subsequent evaporation [9] in the presence of massless fermions that carry the same global charge. One might think, therefore, that, in the absence of a viable mechanism that could naturally produce very large solitons, no relic Q-balls could remain in the present universe.

In this paper, we show that there exists an effective mechanism for the production of Q-balls with enormous charges, big enough to survive until present. The basic idea is the following. A complex scalar field $\varphi$ inside the Q-ball is in a coherent state with timedependent phase: $\varphi=R(x) e^{i \omega t}$, where the radial component $R(r), r=\sqrt{\vec{x}^{2}}$, tends to zero as $r \rightarrow \infty$. An infinite size Q-ball with $R(r)=$ const, Q-matter [2], is similar in nature to the coherent states of a scalar condensate often encountered in inflationary cosmology. For example, formation of the scalar condensate with non-zero baryon number is the starting 
point for the Affleck-Dine scenario for baryogenesis [10]. In this scenario, a combination of squarks and sleptons, or some other fields carrying a baryon or lepton number, has a large expectation value along some flat direction of the potential at the end of inflation. At large VEV, the baryon number can be strongly violated by the high-scale physics. As a result of the baryon number non-conservation, along with the $\mathrm{CP}$ violation, the scalar condensate acquires a baryon number. The subsequent evolution leads it into the domain of conserved baryon number. From this point on, it can be thought of as Q-matter.

Depending on the dynamics, an initially spatially-homogeneous scalar condensate that carries a conserved $\mathrm{U}(1)$ charge may become unstable with respect to small coordinatedependent perturbations and develop a spatial pattern that comprises domains of high and low charge density. If a theory admits non-topological solitons [1, 2], the lowest energy state in a given charge sector is a Q-ball. Very large (and, thus, stable) Q-balls can, in fact, be produced this way.

A related issue is that of the string moduli. If their evolution leads to formation of isolated solitons, it can help alleviate the cosmological moduli problem [11, 12]. The weaklyinteracting moduli may possess some energy density during or after the nucleosynthesis. However, if this energy density is incarcerated inside Q-balls, it may be harmless.

In what follows, we first discuss the stability and decay of Q-balls. Then we analyze formation of Q-balls through solitosynthesis and collisions. We then consider the evolution of a complex scalar field and describe the formation of a spatial pattern that can evolve into Q-balls. Finally, we discuss the contribution of relic Q-balls to dark matter and comment on the possibility of solving the cosmological moduli problem through the incarceration of malicious moduli.

\section{Lifetime of Q-balls}

The fate of Q-balls in the early universe is ultimately determined by their lifetime. If the soliton in question is made of a scalar that has no interactions with light fermions, then it is entirely stable. If such objects had formed through the breakdown of a homogeneous condensate, they would have survived until the present and would contribute to the matter density of the universe as a form of dark matter. 
If, however, the scalar particles can decay into lighter fermions that carry the same global charges, as is the case with the squarks and sleptons of the MSSM, then it is the evaporation rate that determines the lifetime of a Q-ball [9]. Clearly, it is only the fermionic decay modes that ought to be considered in this respect, because, in the sector of scalar degrees of freedom, the Q-ball is the state of minimal energy, and it cannot decay into bosons.

Depending on the scalar potential $U\left(\varphi_{1}, \ldots, \varphi_{n}\right)$, the mass of a large Q-ball may grow with charge as $m_{Q} \sim Q^{p}$, where $0<p<1$. If the quantity

$$
\frac{U\left(\varphi_{1}, \ldots, \varphi_{n}\right)}{\sum q_{i} \varphi_{i}^{2}}
$$

where $q_{i}$ is the charge of the field $\varphi_{i}$ (some of the charges may be zero), is minimized at a finite value of $\varphi$, then the mass of a large Q-ball is proportional to the first power of $Q$ [2]. In this case the mass per unit charge does not depend on the size of the Q-ball.

However, if $U(\varphi)$ is essentially flat, i.e. if it grows slower than the second power of $\varphi$, then, in general, $p<1$ [13]. Such potentials can arise naturally in theories with supersymmetry breaking communicated at low energy, for example, by gauge interaction (for review, see, e.g., Ref. [14] and references therein). If the potential is essentially flat for large $\varphi \gg m_{S}$, then $m_{Q} \sim Q^{3 / 4}$ [13]. We recapitulate briefly the argument of Ref. [13]. The field inside the Q-ball is $\varphi_{i}(x, t)=\varphi_{i}(x) \exp \left\{i q_{i} \omega t\right\}$ [3]. The value of $\omega$ is determined by extremizing the functional

$$
\mathcal{E}_{\omega}=\int d^{3} x\left[\frac{1}{2} \sum_{k}\left|\nabla \varphi_{k}\right|^{2}+\hat{U}_{\omega}(\varphi)\right]+\omega Q,
$$

where $\hat{U}_{\omega}(\varphi)=U(\varphi)-\frac{1}{2} \omega^{2} \sum_{k} q_{k}^{2}\left|\varphi_{k}\right|^{2}$. Written in terms of dimensionless variables $\xi=\omega x$ and $\psi_{i}=\varphi_{i} / \omega, \mathcal{E}_{\omega} \approx a \omega+b / \omega^{3}+\omega Q$, if $U(\varphi) \approx$ const. Here $a$ and $b$ are independent of $\omega$. Therefore, the extremum of functional (2) is achieved for $\omega \propto Q^{1 / 4}$, which corresponds to

$$
m_{Q} \propto Q^{3 / 4}
$$

By virtue of the relation (3), a sufficiently large Q-ball is entirely stable against decay into all but massless fermions because its energy per unit charge can be less than the mass of the lightest fermion. In the MSSM, there are Q-balls (B-balls) made of squarks and Higgs fields [3], for which the baryon number plays the role of the $\mathrm{U}(1)$ charge discussed above. 
The lightest baryons, nucleons, have mass of order $m_{n} \sim 1 \mathrm{GeV}$. A B-ball is entirely stable if it has charge

$$
Q \equiv B \gtrsim\left(\frac{m_{\varphi}}{m_{n}}\right)^{4} \sim 10^{8}
$$

where we took $m_{\varphi} \sim 10^{2} \mathrm{GeV}$ for the squark mass.

If the Q-ball has a lepton charge, but no baryon charge, then it can evaporate [9] by emitting lepton number from the surface with (nearly massless) neutrinos. It is beyond the scope of the present work to evaluate the decay width of slepton Q-balls in the MSSM taking into account the variety of fermionic decay modes. Instead, we will obtain a rough estimate by adopting the discussion of Ref. [9] to our case.

The model of Ref. 9] had a Yukawa coupling of a scalar field $\varphi$ to massless fermions. Inside the Q-ball, the Dirac sea of fermions is filled; and the Fermi pressure inhibits further decay. The decay of the scalar condensate can, therefore, proceed through the surface only, and its rate is suppressed by the surface-to-volume ratio for large Q-balls. The rate of such decay is [9]

$$
\frac{d Q}{d A d t}<\frac{\omega^{3}}{192 \pi^{2}}
$$

where $A$ is a Q-ball surface area. For the lifetime of an L-ball to exceed the age of the Universe, its charge should be $Q>10^{32}$.

The effects of gravity on Q-balls are very small and will be neglected, as long as the size of the soliton is much larger than the corresponding Schwartzschild radius. For a Q-ball in a flat potential, this is the case if $Q \ll Q_{g}=\left(m_{P} / m_{\varphi}\right)^{4} \sim 10^{64}$ for $m_{\varphi} \sim 100 \mathrm{GeV}$. A Q-ball with charge that exceeds $Q_{g}$ would be a black hole. It would evaporate through Hawking radiation, and its global charge would be lost. In our analyses we never encounter Q-balls with such large charges, and we don't know of any mechanism by means of which they could form.

If the potential is not flat, then $E_{Q} \sim Q$ [1, 2], and the Q-ball would decay through evaporation [9] regardless of the value of its charge. (The charge needed to survive the evaporation would exceed the black hole limit by many orders of magnitude.)

At finite temperature, the existence of Q-balls depends on the form of the temperaturedependent effective potential. If it admits Q-balls, then the thermal processes do not affect an 
existing soliton. If, however, a Q-ball is formed out of equilibrium at some temperature $T$ in a system that does not allow Q-ball solutions, the collisions with particles in the hot plasma can lead to an erosion of the condensate. For large solitons this process is strongly suppressed by the small penetration depth of the outside particles inside the Q-ball. A particle $\psi$ that interacts with the field $\varphi$ receives a contribution to its mass of order $m_{\psi}(x) \sim g\langle\varphi(x)\rangle$ inside the Q-ball; outside Q-ball $m_{\psi} \sim g T$. At temperature $T$ a $\psi$ particle cannot penetrate inside the Q-ball beyond the point $x$ where $\varphi(x) \sim T / g$. The condensate can be thought of as a coherent state of $\varphi$ particles with mass $m_{\text {cond }} \sim m_{\varphi} / Q^{1 / 4}$ and density $\omega \varphi^{2}(x)$. If a Q-ball is created at the time $t_{i}$, the total number of collisions with the $\psi$ particles in plasma during the lifetime of the cooling universe is

$$
\int_{r_{0}}^{\infty} r^{2} d r \int_{t_{i}}^{\infty} d t T^{3}(t) \sigma \omega \varphi^{2}(r) v_{\psi}(r)
$$

where $\sigma \sim g^{2} / T^{2}$ is a cross-section of particle-particle collision, the velocity of the $\psi$ particle at a given point is determined by energy conservation. The stopping point, $r_{0}$ is defined by the relation $m_{\psi}(r)=T$, or $g \varphi(x)=T$. For squark B-balls $g \sim 1$. One can use $T^{2}=M_{0} / t$ to relate temperature to time in a

radiation dominated universe. Here and below $M_{0}=M_{P} /\left(1.66 n_{\text {eff }}^{1 / 2}\right)$, and $n_{\text {eff }}$ is the number of effective degrees of freedom. In each collision, only a fraction of energy, $\delta E \sim$ $T\left(m_{\text {cond }} / m_{\psi}\right)^{2}$, is transmitted to the condensate. Therefore, using (6), the total energy transmitted to the Q-ball is

$$
\Delta E \sim R^{2} m_{\text {cond }} \omega M_{0} T^{2} \sigma \int_{r_{0}}^{\infty} \frac{\varphi^{2}(r) v(r)}{m_{\psi}(r)} d r .
$$

This is sufficient to knock out $\Delta Q \sim \Delta E / m_{\varphi}$ particles from the condensate. For a "flat" potential,

$$
\Delta Q \sim\left(\frac{T}{m_{\varphi}}\right)^{2}\left(\frac{M_{0}}{m_{\varphi}}\right) .
$$

A Q-ball can survive the erosion by thermal plasma if $\Delta Q<Q$.

\section{Solitosynthesis and collisions}

Solitosynthesis of non-topological solitons [5, 6, 7] is the process of charge accretion that may take place in a system at finite temperature with non-zero charge asymmetry $\eta$. Q-ball 
is the minimum of energy $E$ in the sector of fixed charge, but is also a state of small entropy $S$ (because it is an extended object). For high temperature it may not be the minimum of free energy $F=E-T S$ because of the $T S$ term. At some small enough temperature $T_{0} \sim m_{\varphi} /|\ln \eta|$, however, the second term in $F$ is no longer important, and the gain from minimizing $E$ overwhelms the loss from lowering the entropy [5, 6, 7]. At that point, Q-ball is the minimum of free energy. In the absence of light charged fermions, a copious production of Q-balls can take place.

However, if there are light fermions carrying the same global charge as the scalars, then the charge asymmetry in the state of the lowest free energy is accommodated entirely in the fermionic sector, thus making solitosynthesis impossible. For example, the solitosynthesis of baryonic and leptonic Q-balls in the MSSM is only possible in a B or L breaking minimum of the potential, where the quarks or leptons are sufficiently massive [7].

Even in the absence of light charged fermions, solitosynthesis is unlikely to produce large Q-balls unless the charge asymmetry is very large [5, 6]. The baryon asymmetry of the universe is as small as $10^{-10}$, which is certainly not sufficient for synthesizing large enough B-balls to satisfy the stability bound (ब1). One can ask whether the subsequent mergers of small Q-balls can increase the average size considerably.

Regardless of the mechanism that lead to the formation of Q-balls, their size can further change because of the collisions in which two Q-balls would merge and form a larger soliton. For simplicity we will neglect the evaporation of Q-balls assuming that they are made of stable scalars. The effect of collisions is described by the kinetic equation

$$
\frac{\partial}{\partial t} N_{Q}+3 H N_{Q}=\left[\frac{1}{2} \int\left\langle\sigma_{Q} v\right\rangle N_{Q-q} N_{q} d q-N_{Q} \int\left\langle\sigma_{Q} v\right\rangle N_{q} d q\right],
$$

where $N_{Q}$ is the density of Q-balls with charge $Q$, and $H$ is the Hubble constant. We note in passing that if the individual particles are included as $Q=1$ objects, the same equation (9) can describe the charge accretion via absorption of particles by Q-balls. The cross-section for the merger of two Q-balls is determined mainly by their geometrical size, $\sigma_{Q} \sim 4 \pi R_{Q}^{2}$, and the average velocity of their non-relativistic Brownian motion is $v \sim \sqrt{T / m_{Q}}$. For a thick-wall Q-ball in a flat potential the quantity $\left\langle\sigma_{Q} v\right\rangle \propto R_{Q}^{2} / \sqrt{m_{Q}}$ changes with charge very slowly, as $Q^{1 / 8}$. Therefore, for a large range of charges, it can be considered constant. One 
can define the reduced number density of Q-balls as $n_{Q}=N_{Q} / T^{3}$. Then

$$
\frac{\partial}{\partial \tau} n_{Q}=\int n_{Q} n_{Q-q} d q-2 n_{Q} \int n_{q} d q
$$

where $\tau=\tau_{\max }\left[1-\left(T / T_{0}\right)^{3 / 2}\right]$ and varies from $\tau=0$ to $\tau_{\max }=M_{0}\left\langle\sigma_{Q}\right\rangle T^{3 / 2} / \sqrt{m_{Q}}$. This equation can be solved by applying a Laplace transform to disentangle the integrals on the right-hand side. The resulting equation is a first-order differential equation for $f_{s}(t) \equiv$ $\int_{0}^{\infty} n_{Q} \exp (-Q s) d Q$,

$$
\frac{\partial}{\partial \tau} f_{s}=f_{s}^{2}-2 f_{s} f_{0}
$$

Let us consider, for example, an initial distribution that peaks near $Q=Q_{0}$. The value of $Q_{0}$ can be set by some earlier process, for example, solitosynthesis. We require that at $\tau=0$, $n_{Q}(1)=\epsilon \delta\left(Q-Q_{0}\right)$, and, hence, $f_{s}(1)=\epsilon \exp \left\{-Q_{0} s\right\}$. This initial condition corresponds to a set of equal size Q-balls with charge $Q_{0}$ each and number density $\epsilon$ that start merging through collisions at $\tau=0$. The solution is

$$
f_{s}(\tau)=\frac{\epsilon}{(1+\epsilon \tau)^{2}} \frac{1}{e^{Q_{0} s}-\frac{\epsilon \tau}{1+\epsilon \tau}} .
$$

The inverse Laplace transform of $f_{s}(\tau)$ in equation (12) is a contour integral that can be found by summing over the residues of the function $\left(f_{s}(\tau) \exp \{Q s\}\right)$ in the complex plane.

$$
n_{Q}=\frac{\epsilon}{(1+\epsilon \tau)^{2}}\left[\frac{\epsilon \tau}{1+\epsilon \tau}\right]^{\left(Q-Q_{0}\right) / Q_{0}} \sum_{n} \delta\left(Q / Q_{0}-n\right)
$$

where $n$ is an integer. The distribution (13) describes an array of delta-functions at integer values of $Q / Q_{0}$ weighted differently for different $\tau$. This is to be expected because each collision changes the charge of a Q-ball by an integer multiple of $Q_{0}$.

As temperature goes to zero, $\tau \rightarrow \tau_{\max }$, the distribution of Q-balls approaches (for $\left.\epsilon \tau_{\max } \gg 1\right)$

$$
\lim _{\tau \rightarrow \tau_{\max }} n_{Q}(t) \propto \frac{\epsilon}{\left(\epsilon \tau_{\max }\right)^{2}} \exp \left(-\frac{1}{\epsilon \tau_{\max }} \frac{Q}{Q_{0}}\right)
$$

for integer $Q / Q_{0}$. This distribution is suppressed for large values of $Q / Q_{0} \gg \epsilon \tau_{\max }$.

This process, which, for reasons explained earlier, is only relevant for weakly interacting stable particles, may considerably increase the average size of a Q-ball. 


\section{$3 \quad$ Instability and pattern formation in the motion of a scalar condensate}

We now turn to the mechanism that can, in fact, lead to the formation of very large Qballs in the early universe. In particular, huge Q-balls made of squarks and sleptons can be produced this way.

Evolution of a spatially homogeneous condensate carrying a baryon or lepton number has been studied in detail in connection with the Affleck-Dine [10] mechanism for baryogenesis. The scalar under consideration is a complex field that carries some $\mathrm{U}(1)$ charge $(e . g$., the baryon number) and can be a combination of squarks and sleptons of the MSSM [15]. If the $\mathrm{U}(1)$ charge is preserved by the low-energy physics, the eventual decay of such condensate can contribute to the corresponding charge asymmetry.

It is possible, however, that the spatially homogeneous condensate develops an instability that leads to the formation of domains with higher and lower charge density. If the theory admits Q-balls, such pattern can evolve into Q-balls before the condensate is eroded by decay. Thermal fluctuations cannot wash out the coherent state as long as the scalar VEV is larger than $g T$, where $g$ is a typical coupling. This is because all the particles coupled to $\varphi$ with a coupling $g$ have masses of order $g\langle\varphi\rangle \gg T$. After the Q-balls form, the subsequent decay of the condensate may become impossible (if the Q-ball energy per unit charge is less than the mass of the lightest Q-charged fermion) or slow (if the charge is sufficiently large). The relic Q-balls can thus survive until present and contribute to the dark matter in the universe.

Under very general conditions the scalar condensate can be described by the classical equations of motion in the effective potential with initial conditions determined by the highscale physics or inflation. It is convenient to write the complex field $\phi=R e^{i \Omega}$ in terms of its radial component and a phase, both real functions of the space-time coordinates. We are interested in the evolution of the scalar field in the low-energy domain, where the baryon number violating processes are suppressed, and we will assume that the scalar potential preserves the $\mathrm{U}(1)$ symmetry: $U(\varphi)=U(R)$, where $U(R)$ may depend on time explicitly. The classical equations of motion in the spherically symmetric metric $d s^{2}=d t^{2}-a(t)^{2} d r^{2}$ are 


$$
\begin{aligned}
\ddot{\Omega}+3 H \dot{\Omega}-\frac{1}{a^{2}(t)} \Delta \Omega+\frac{2 \dot{R}}{R} \dot{\Omega}-\frac{2}{a^{2}(t) R}\left(\partial_{i} \Omega\right)\left(\partial^{i} R\right) & =0, \\
\ddot{R}+3 H \dot{R}-\frac{1}{a^{2}(t)} \Delta R-\dot{\Omega}^{2} R+\frac{1}{a^{2}(t)}\left(\partial_{i} \Omega\right)^{2} R+(\partial U / \partial R) & =0,
\end{aligned}
$$

where dots denote the time derivatives, and the space coordinates are labeled by the Latin indices that run from 1 to 3 . The Hubble constant $H=\dot{a} / a$, where $a(t)$ is the scale factor, is equal to $t^{2 / 3}$ or $t^{1 / 2}$ for the matter or radiation dominated universe, respectively.

A spatially homogeneous solution of equations (15) and (16) has vanishing gradients of $R$ and $\Omega$. If one neglects the expansion of the universe $(H=0)$ and if $\dot{R}=0$, the solution describes a universe filled with Q-matter [2]. If the theory admits Q-balls, Q-matter can become unstable with respect to its break-up into Q-balls. In a static universe, the conditions of stability were analyzed in Ref. [16]. The results cannot be applied directly to the case of the expanding universe, where the equations of motion are time-dependent and the red shift of linear perturbations is important. Under some conditions spatial inhomogeneities may destabilize a given solution of equations (15) and (16).

From the equations of motion (15) and (16), one can derive the equations for small perturbations $\delta \Omega$ and $\delta R$ :

$$
\begin{gathered}
\ddot{\delta \Omega}+3 H(\dot{\delta \Omega})-\frac{1}{a^{2}(t)} \Delta(\delta \Omega)+\frac{2 \dot{R}}{R}(\dot{\delta} \dot{\Omega})+\frac{2 \dot{\Omega}}{R}(\dot{\delta R})-\frac{2 \dot{R} \dot{\Omega}}{R^{2}} \delta R=0 \\
\ddot{\delta R}+3 H(\dot{\delta} R)-\frac{1}{a^{2}(t)} \Delta(\delta R)-2 R \dot{\Omega}(\dot{\delta} \dot{\Omega})+U^{\prime \prime} \delta R-\dot{\Omega}^{2} \delta R=0 .
\end{gathered}
$$

In general, the analysis of instabilities must be done numerically. However, one can identify the growing modes and estimate the length scale of the resulting pattern by using a WKB approximation. This approximation is valid as long as the background solution varies slowly with time.

To examine the stability of a homogeneous solution $\varphi(x, t)=\varphi(t) \equiv R(t) e^{i \Omega(t)}$, let us consider a perturbation $\delta R, \delta \Omega \propto e^{S(t)-i \vec{k} \vec{x}}$ and look for growing modes, $\operatorname{Re} \alpha>0$, where $\alpha=d S / d t$. The value of $k$ is the spectral index in the comoving frame and is red-shifted with respect to the physical wavenumber $\tilde{k}=k / a(t)$ in the expanding background. Of course, 
if the instability develops, the linear approximation is no longer valid. However, we assume that the wavelength of the fastest-growing mode sets the scale for the high and low density domains that evolve into Q-ball eventually. This assumption can be verified post factum by comparison with a numerical solution of the corresponding partial differential equations (15) and (16), where both large and small perturbations are taken into account.

The dispersion relation follows from the equations of motion:

$$
\left[\alpha^{2}+3 H \alpha+\frac{k^{2}}{a^{2}}+\frac{2 \dot{R}}{R} \alpha\right]\left[\alpha^{2}+3 H \alpha+\frac{k^{2}}{a^{2}}-\dot{\Omega}^{2}+U^{\prime \prime}(R)\right]+4 \dot{\Omega}^{2}\left[\alpha-\frac{\dot{R}}{R}\right] \alpha=0
$$

If $\left(\dot{\Omega}^{2}-U^{\prime \prime}(R)\right)>0$, there is a band of growing modes that lies between the two zeros of $\alpha(k), 0<k<k_{\max }$, where

$$
k_{\max }(t)=a(t) \sqrt{\dot{\Omega}^{2}-U^{\prime \prime}(R)} .
$$

If $k_{\max }(t)$ defined by relation (20) is constant or growing with time, then each mode has an unlimited time to develop and the size of a resulting inhomogeneity in the comoving frame is determined by the value of $k$ that corresponds to the maximum of $\alpha$.

If, on the other hand, $k_{\max }(t)$ decreases with time, then the modes may not have the time to grow sufficiently before they are red-shifted from the resonance. A constant or growing with time width of the band (20) is a sufficient (although not necessary) condition for the instability.

Quantitatively, the amplification of a given mode $k$ is characterized by the exponential of

$$
S(k)=\int \alpha(k, t) d t
$$

We look for the value of the best-amplified mode with wavenumber $k_{\text {best }}$ that maximizes $S(k)$. The instability develops when $e^{S\left(k_{\text {best }}\right)} \gg 1$. The amount of amplification needed in a given system is determined by the size of the fluctuations. These can be thermal, or of a different origin (see, e. g., the discussion in Ref.[17]). The critical value of $S\left(k_{\text {best }}\right) \sim \ln (R / \delta R)$ signals the non-linear regime and corresponds to the breakdown of the homogeneous solution.

As an example, let us consider the following potential: 


$$
U(\varphi)=m_{S}^{4} \ln \left(1+\frac{\varphi^{\dagger} \varphi}{m_{S}^{2}}\right)-c H^{2} \varphi^{\dagger} \varphi+\frac{\lambda^{2}}{m_{P}^{2}}\left(\varphi^{\dagger} \varphi\right)^{3},
$$

where $c$ and $\lambda$ are some constants and $H \sim 1 / t$. Such potential can arise in theories with supersymmetry breaking communicated at the scale $m_{S} \ll m_{P}$ [18, 19] and is similar to those discussed in Ref. [15, 19] in connection with baryogenesis. The mass term proportional to the Hubble constant appears naturally from the Kähler potential as $\int d^{4} \theta\left(\left\langle\chi^{\dagger} \chi\right\rangle / m_{P}^{2}\right) \varphi^{\dagger} \varphi$ [12], where $\chi$ is some field with non-zero density, whose F-component has a VEV. This term essentially characterizes supersymmetry breaking by the finite energy density that dominates the universe at time $t$.

We will assume that the universe before Q-balls formation is matter dominated. For large $|\varphi|$, the logarithmic term in (22) can be neglected and the equations of motion admit an adiabatic solution for $R(t) \sim 1 / t^{1 / 2}$ and $\omega \equiv \dot{\Omega} \sim 1 / t$, that follows closely the location of the minimum of $U$. The conservation of charge per comoving volume implies $\dot{\Omega}(t) R^{2}(t) a(t)^{3}=$ const.

It is clear from equation (20) that the band of resonance, bounded from above by $k_{\max } \sim$ $t^{-1 / 3}$, becomes narrower with time. In other words, each mode in the amplification region is red-shifted faster than $k_{\max }$, and the amplification may not be sufficient. We note that the time dependence of $\omega$ is the same as that of $H$, both are inversely proportional to time.

For small $|\varphi| \sim m_{S}$, the first term in equation (22) dominates: $U(\varphi) \approx m_{S}^{2} \varphi^{\dagger} \varphi$. In this case, the equation of motion for $R$ is linear and its solution is $\varphi \sim 1 / t$, with a constant $\omega=m_{S}$. Clearly, in this regime there is an unlimited growth of all modes in the band of instability because $k_{\max } \sim a(t)$ is growing with time and the band widens.

Qualitatively, the behavior of $\omega$ is shown in Fig. 11. As long as the logarithmic term in the potential can be neglected, $\omega$ changes as $1 / t$, roughly, at the rate of the expansion of the universe, and its value is of order $H$. However, at later times $\omega$ approaches a plateau $\omega \sim m_{S}$. A characteristic length of growing instability is determined by the fastest-growing mode at the time when the rate of change in $\omega$ becomes different from $1 / t$.

To estimate a charge of Q-balls produced we assume that Q-matter was formed at some time $t_{0}$ with charge density $q_{0} \equiv q\left(t_{0}\right)=\omega_{0} \varphi_{0}^{2}$. In a matter dominated universe $q(t)=$ $q_{0}\left(t_{0} / t\right)^{2}$. Naturally, the wavelength $1 / k \sim \chi / H$ of the best-amplified mode cannot exceed the size of the horizon at the time $t_{i}$ when the instability develops, $\chi<1$. The total charge 


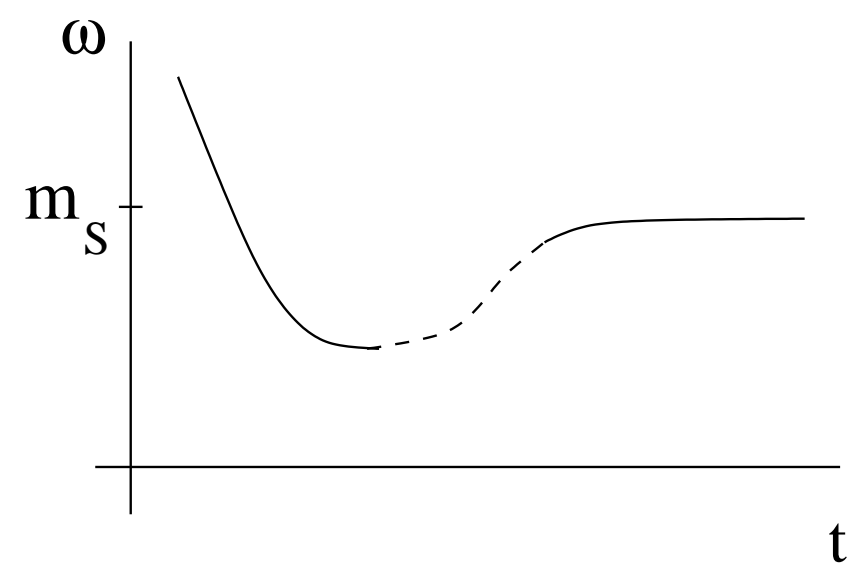

Figure 1: At early times $\omega$ changes at, roughly, the same rate as the expansion of the universe. All modes of instability are red-shifted before they can grow sufficiently. However, at later times $\omega$ approaches a constant value $\sim m_{S}$ and the instabilities develop. The size of a domain is determined by the fastest-growing mode at the time when the rate of change in $\omega$ becomes different from $1 / t$.

of Q-matter inside the part of horison corresponding to the best amplified model gives an estimate for the charge of Q-ball:

$$
Q \sim q_{0} t_{0}^{2} t_{i} \chi^{3} .
$$

The time $t_{i}$ and parameter $\chi$ are to be found from numerical calculations. We performed them for a potential (22) for different sets of parameters. The amplification $S(k)$ of a given mode was found through numerical integration. We took the initial time of the Q-matter formation to be $t_{0}=10^{-2} \mathrm{GeV}^{-1}$. For $\lambda=1 / 2, c=1, m_{S}=10^{4} \mathrm{GeV}$ and $q_{0}=10^{24} \mathrm{GeV}^{3}$ (this corresponds to $\varphi_{0}=2.5 \times 10^{10} \mathrm{GeV}$ and $\omega_{0}=1.6 \times 10^{3} \mathrm{GeV}$ ) we observed that the best-amplified mode was two orders of magnitude smaller than the horizon size, $\chi \simeq 10^{-2}$, at the time when the instability developed $\left(S_{\max }(k) \simeq 30\right.$ at $\left.t_{i} \simeq 150 \mathrm{GeV}^{-1}\right)$. This corresponds to the average charge of emerging Q-balls of order $10^{16}$. For a different set of parameters, $m_{S}=10^{2} \mathrm{GeV}, \lambda=0.5 \times 10^{-3}$ and $q_{0}=10^{25} \mathrm{GeV}^{3}$ (which correspond to $\varphi_{0}=2.8 \times 10^{11}$ $\mathrm{GeV}$ and $\omega_{0}=1.3 \times 10^{2} \mathrm{GeV}$ ), we obtain $t_{i} \simeq 1.5 \times 10^{5} \mathrm{GeV}^{-1}, \chi \simeq 10^{-2}$ and $Q \sim 10^{20}$. The band of unstable modes is shown in Figure 2. Of course, the size of Q-balls depends on a model. We have demonstrated, however, that very large Q-balls can be born from an initially homogeneous scalar condensate. In particular, the flatter, than logarithmic, potentials produce even larger Q-balls. It would be desirable to study the instabilities beyond the linear analyses performed so far, when the solution is significantly different from the 


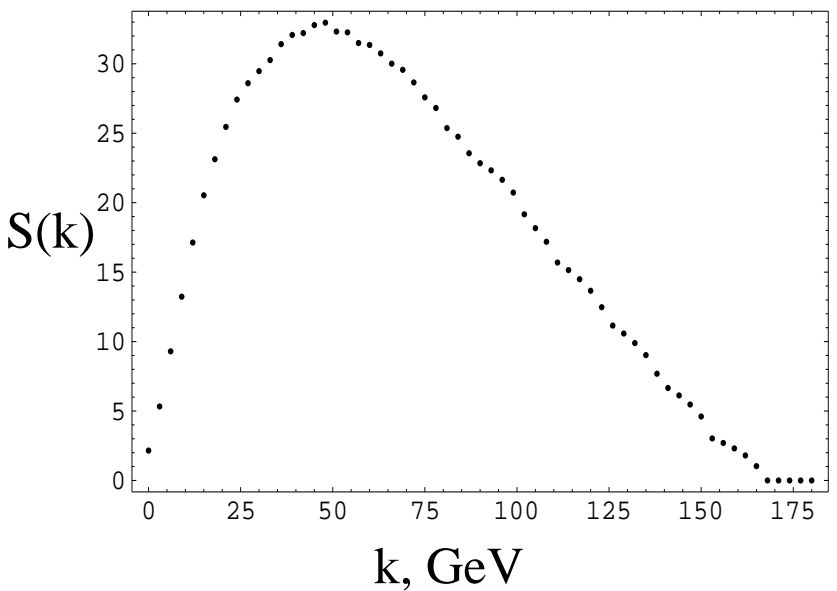

Figure 2: The amplification $S(k)=\int_{t_{0}}^{t_{\max }} \alpha(k, t) d t$ of growing modes is computed numerically for different values of $k$. The fastest-growing mode has the wave number $k \sim 10^{2} H$ at the time when the instability develops. An individual Q-ball gathers charge from, roughly, $10^{-2}$ of the size of the horizon.

original homogeneous one, as illustrated in Figure 3. We leave this for future work.

\section{Surviving Q-balls as dark matter}

It is beyond the scope of this paper to examine in detail the implications of solitonic dark matter formed from the breakdown of a scalar condensate. We will limit our discussion to a few general remarks.

We have seen that B-balls formed through the instability can easily satisfy the bound (田). If finite temperature effective action admits B-balls, they will survive till present. If not, they may or may not be eroded by the thermal plasma, depending on the model parameters and temperature $T_{i}$ at the time of the Q-ball formation. From equation (8) they are not destroyed if $T_{i}<m_{\varphi}\left(m_{\varphi} Q / M_{0}\right)^{\frac{1}{2}}$. Surviving B-balls would contribute to the dark matter in the Universe. The amount of the dark matter is determined by the efficiency of the conversion process that leads from a uniform condensate to B-balls. As the instability develops, part of the charge (baryon number) stored in the condensate can be lost in decay and give rise to the baryonic matter. It seems reasonable to assume that the amount of baryonic charge locked inside the Q-balls can be roughly comparable (within a few orders of magnitude) to the amount of charge that leaked out with individual nucleons.

If the scalar condensate that evolves into Q-balls has a lepton charge, but no baryon 


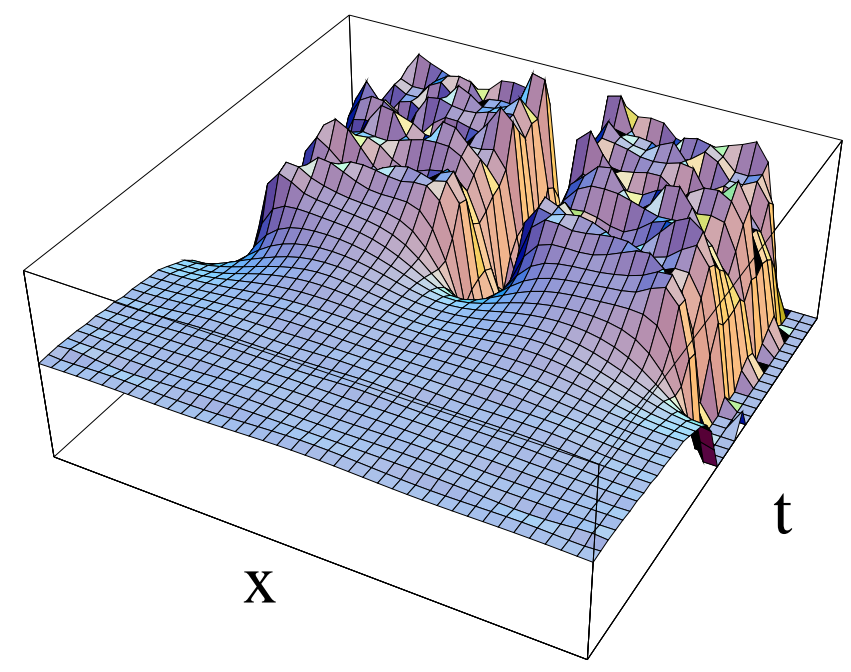

Figure 3: The charge density per comoving volume in $(1+1)$ dimensions for a sample potential analyzed numerically during the period when the spatially homogeneous condensate breaks up into high- and low-density domains. Two domains with high charge density are expected to form Q-balls.

charge, then a Q-ball can evaporate [9] by emitting lepton number from the surface with (nearly massless) neutrinos. We have seen that, for the lifetime of an L-ball to exceed the age of the Universe, its charge should be $Q>10^{32}$ according to equation (5). Smaller L-balls must have evaporated by now. Decay of the L-balls during or after the nucleosynthesis may have important ramifications as it would lead to the entropy increase and, if it happens at later times, could distort the spectrum of the cosmic microwave background radiation. This may lead to constraints on the parameters of the MSSM, as well as on the initial conditions after inflation.

Our discussion has bearing on the issue of the so called moduli problem [11, 12]. Small couplings to matter fields are typical for the moduli fields of string theory. This poses a serious problem for cosmology since the energy density carried by the coherent motion of the moduli condensates does not decay until after the nucleosynthesis. The products of such decay can over-produce the entropy, as well as destroy the nuclei. The mechanism we have described may help alleviate the moduli problem by incarcerating moduli inside slowly evaporating Q-balls. On the one hand, this slows down the decay, thus allowing for the moduli Q-balls to still be present in the Universe and contribute to dark matter. On the other hand, whatever decay would have taken place, the detrimental effect of the energetic 
photons on the nuclei is confined to the close vicinity of Q-balls separated by some vast unaffected areas, where the standard nucleosynthesis can take place.

Let us suppose that in the present Universe there is, roughly, the same (to within a few orders of magnitude) baryon number in baryonic Q-balls and in ordinary matter. According to our estimates, the charge of an individual stable B-ball may be from $10^{8}$ to $10^{20}$ or more; and the corresponding number density of Q-balls is then $10^{-20}$ to $10^{-8}$ per nucleon. Perhaps, even much larger Q-balls may emerge from the scalar condensate in models different from ours. The larger Q-balls from this range may be difficult to detect because they are extremely rare. However, the possibility of smaller, and, hence, more populous B-balls as a dark matter candidate seems to be very appealing from an experimental point of view. Some of the heavy relic Q-balls, attracted by the gravitational fields of stars and planets, can sink to the center and remain there. It is conceivable that the deep interior of the small planets might become accessible for exploration in the future and reveal storages of primordial Q-balls. Other solitons may be present in the interstellar medium. However, since their number density is expected to be very small, detection of such objects seems to be a great challenge to the experimentalists' ingenuity. It is also of interest to understand the effect of large Q-balls on the structure formation in the early universe.

We thank G. Dvali, H. Murayama and P. Tinyakov for helpful discussions.

\section{References}

[1] For review, see T. D. Lee and Y. Pang, Phys. Rep. 221 (1992) 251, and references therein.

[2] S. Coleman, Nucl. Phys. B262 (1985) 263.

[3] A. Kusenko, Phys. Lett. B405 (1997) 108.

[4] J. A. Frieman, G. B. Gelmini, M. Gleiser and E. W. Kolb, Phys. Rev. Lett. 60 (1988) 2101; K. Griest, E. W. Kolb and A. Maassarotti, Phys. Rev. D40 (1989) 3529; J. Ellis, K. Enqvist, D. V. Nanopoulos and K. A. Olive, Phys. Lett. B225 (1989) 313.

[5] J. A. Frieman, A. V. Olinto, M. Gleiser and C. Alcock, Phys. Rev. D40 (1989) 3241. 
[6] K. Griest and E. W. Kolb, Phys. Rev. D40 (1989) 3231.

[7] A. Kusenko, Phys. Lett. B406 (1997) 26.

[8] A. Kusenko, Phys. Lett. B404 (1997) 285.

[9] A. Cohen, S. Coleman, H. Georgi and A. Manohar, Nucl. Phys. B272 (1986) 301.

[10] I. Affleck and M. Dine, Nucl. Phys. B249 (1985) 361.

[11] G. Coughlan, W. Fishler, E. Kolb, S. Raby and G. Ross, Phys. Lett. B131 (1983) 59; J. Ellis, D. V. Nanopoulos and M. Quirós, Phys. Lett. B174 (1986) 176; R. de Carlos, J. A. Casas, F. Quevedo and E. Roulet, Phys. Lett. B318 (1993) 447; T. Banks, D. Kaplan and A. Nelson, Phys. Rev. D49 (1994) 779; L. Randall and S. Thomas, Nucl. Phys. B449 (1995) 229; M. Dine, L. Randall and S. Thomas, Phys. Rev. Lett. 75 (1995) 398; T. Banks, M.Berkooz and P. J. Steinhardt Phys. Rev. D52 (1995) 705; A. Linde, Phys. Rev. D53 (1996) 4129;

[12] G. Dvali (hep-ph/9503259).

[13] G. Dvali, A. Kusenko and M. Shaposhnikov (hep-ph/9707423).

[14] C. Kolda, talk presented at SUSY-97 conference, Philadelphia, May 1997 hepph/9707450).

[15] M. Dine, L. Randall and S. Thomas, Nucl. Phys. B458 (1996) 291.

[16] K. Lee, Phys. Rev. D50 (1994) 5333.

[17] V. Mukhanov, H. Feldman and R. Brandenberger, Phys. Rep. 215 (1992) 203.

[18] G. Dvali, Q. Shafi and R. Schaefer, Phys. Rev. Lett. 73 (1994) 1886; G. Dvali, Phys. Lett. B387 (1996) 471.

[19] A. de Gouvêa, T. Moroi and H. Murayama, Phys. Rev. D56 (1997) 1281. 